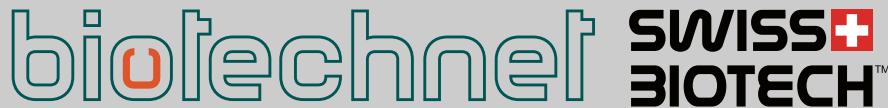
switzerland

\section{Summer School 2017 - Exchange of Ideas for Biologists}

\section{Elsbeth Heinzelmann, Journalist science + technology}

Abstract: When Daniel Gygax, former President of Swiss biotechnet, and Letizia Vittorelli from the University of Palermo had the idea in 2005 to create the Summer School on Advanced Biotechnology, their aim was to exchange ideas and experience in a friendly and open-minded atmosphere to advance scientific knowledge. The network is a recipe for success: In 2017, it was the turn of the HES-SO Valais/Wallis to welcome the event to Sion from September $3-6$.

Keywords: Active antibiotic monitoring - Bacterial biosensors . Fed-batch fermentation - Microalgae $\cdot$ Microbial fermentation

"Knowledge is the only competitive advantage of our times; it grows through open interaction with others." These words of British economist and Nobel laureate Ronald Coase seem to have been the initial spark for the Summer School on Advanced Biotechnology, which today is a pioneer in collaboration and knowledge sharing. Let's pick out a few presentations from the 2017 edition and begin with Functional Genomics.

\section{Identifying Cancer Treatment Options Using Comprehensive Molecular Diagnostics}

At ETH Zurich, the Clinical Bioinformatics Unit of NEXUS Personalized Health Technologies offers customised bioinformatics and statistics services for analysis in the field of biomedical research. For Dr. Daniel Stekhoven, head of this technology platform, high-throughput genomics have changed the way biomedical research is performed: "The transition from directed testing of a few specific targets to analysing comprehensive highthroughput data offers tremendous possibilities, particularly for the diagnosis of patients with rare diseases, for tumours lacking known targetable mutations, or for patients for whom routine diagnostic and treatment paradigms have failed," he states. Establishing comprehensive molecular diagnostics for clinical use requires specific protocols accounting for stringent quality control, privacy issues and computational requirements. "To this end, we - a group of bioinformatics scientists, statisticians and cancer biologists - have developed a workflow for the molecu- lar profiling of matched tumour and normal samples to improve clinical decision support. In order to gain an even more comprehensive understanding of the tumour, we have recently begun to include transcriptomic data in the analysis." Using publicly available transcriptome data as a reference, clinical researchers can assess over-/underexpression of genes of interest and complement genome measurements with gene regulatory data. In addition to the identification of somatic variants, expression changes and gene fusions, their workflow links the detected alterations to possible treatment options. The member of the expert group 'Molecular Oncology' at the University Hospital Zurich concludes: "The analysis results are summarised in a concise and clearly structured clinical report designed to form the basis for discussions in a molecular tumour board at the hospital."

stekhoven@nexus.ethz.ch www.nexus.ethz.ch

\section{Hints from Differential Proteomics for a Rational Yield Improvement of Biomolecule Production by Microbial Fermentation}

"Actinomycetes, Gram-positive filamentous bacteria, are a very prolific source of biological antibiotics", [1] states Dr. Giuseppe Gallo, PhD from the Laboratory of Molecular Microbiology and Biotechnology, Department STEBICEF at University of Palermo. "Indeed, in actinomycetes antibiotic biosynthesis is elicited as an answer to different intracellular and extracellular signals, including cell density and nutrient limitation. ${ }^{[2,3]}$ These stimuli activate a cascade of regulatory events and molecular processes which eventually promote the expression of the genes specifically involved in antibiotic biosynthesis." Products of these genes are involved in the assembly of antibiotic backbone which may require amino acids and/or metabolic intermediates from central carbon metabolism as precursors. "The availability of antibiotic precursors often results in a bottleneck in production yield. Thus, strategies based on strain improvement through metabolic engineering by gene manipulation may be successful," says the Italian scientist. "However, this approach has to take into account the high level of complexity characterising the regulatory network controlling antibiotic biosynthesis. In this view, omics-based approaches can help in understanding the molecular cell physiology of producer strains."

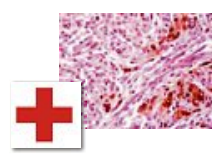

Tumor biopsy and DNA \& RNA extraction

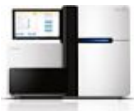

Sequencing
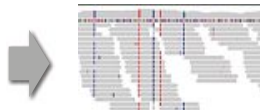

Bioinformatics analyses

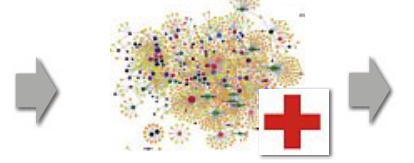

Association of genetic variants with potential treatments

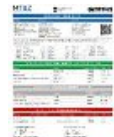

Summarization in concise clinical report

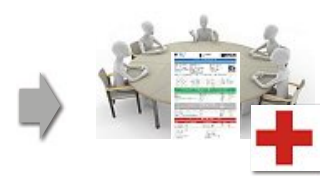

Utilization in molecular tumor board 


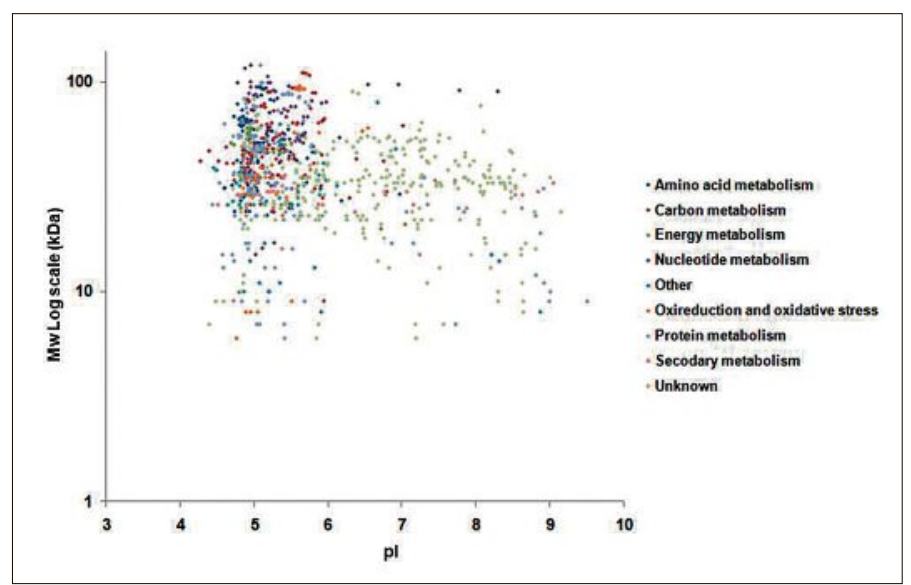

Giuseppe Gallo: Experimental 2D-distribution - based on molecular weight $(\mathrm{Mw})$ and isoelectric point $\mathrm{pl}$ - of differentially regulated protein species associated with NAl-107 production in Microbispora ATCCPTA-5024. Functional classification was performed according to KEGG metabolic database (http://www.genome.jp/kegg/). (Source Giuseppe Gallo)

Microbispora ATCC-PTA-5024 is an actinomycete that produces the promising lantibiotic NAI-107 that was shown to be effective against Gram-positive bacteria - including methicillinresistant Staphylococcus aureus (MRSA), glycopeptide-intermediate $S$. aureus (GISA) and vancomycin-resistant enterococci (VRE) - and some Gram-negative bacteria. Since Microbispora ATCC-PTA-5024 was a poorly characterised strain, its molecular physiology was investigated within a collaborative project financed by the European Commission (contract 245066 for FP7-KBBE-2009-3). In particular, by integration of proteomic, genomic and bioinformatic tools an extensive picture of stimuli, genes and molecular processes thereof involved in NAI-107 production was achieved. ${ }^{[4]}$ To reveal patterns of differentially represented proteins associated with NAI-107 production onset and maintenance, differential proteomic analyses were carried-out on biomass samples collected at several time points before and during NAI-107 production. The 2D-proteome patterns comparatively analysed for quantitative variations revealed a total of 752 differentially abundant protein species which were identified by mass spectrometry-based procedures. Bioinformatics resources, available at KEGG metabolic database web page (http://www. genome.jp/kegg/), allowed the functional classification of regulatory and metabolic proteins. In particular, the $14.5 \%$ of the whole cellular functions (1,661 in total) inferred from Microbispora ATCC-PTA-5024 genome were positively or negatively associated with NAI-107 production revealing that nutritional signals and primary metabolism shift-down stimulate the accumulation of protein components involved in regulatory cascades eventually involving nitrogen and phosphate metabolism, cell wall biosynthesis/maturation, lipid metabolism, osmotic stress response, multi-drug resistance, and NAI-107 biosynthesis and transport. Some insights derived from proteome data, like the stimulating role on physiological differentiation of an identified TetR-like regulator or the role of NAI-107 on membrane stability of producer strain, were eventually confirmed by the characterisation of ad hoc genetically engineered strains. The researcher takes stock as follows: "This study supported the relevance of proteomics in revealing players of antibiotic biosynthesis and regulation in actinomycetes."

giuseppe.gallo@iunipa.it
[1] J. Bérdy. J. Antibiot. 2012, 65, 385.

[2] G. Liu, K. F. Chater, G. Chandra, G. Niu, H. Tan, Microbiol. Mol. Biol. Rev. 2013, 77, 112 .

[3] M. Bibb, Curr. Opin. Microbiol. 2005, 8, 208.

[4] G. Gallo, G. Renzone, E. Palazzotto, P. Maonciardini, S. Arena, T. Faddetta, A. Giardina, R. Alduina, T. Weber, F. Sangiogi, A. Russo, G. Spinelli, M. Sosio, A. Scaloni, A. M. Puglia, BMC Genomics 2016, 17, 42.

\section{In Search of Novel Compounds}

Microalgae as a group of photosynthetic organisms are particularly interesting for researchers: they offer valuable substances and low-cost growth in the presence of light, thereby representing untapped potential. Their activities focus on unsearched biobanks, especially a collection of terrestrial algae: the scientists assume that they would be able to exploit innovative active agents, for instance for the pharmaceutical industry. That's why microalgae are a research focus at the MCI - Management Center Innsbruck. Here, scientists such as Maren Kruus, project assistant in the group of Professor Christoph Griesbeck, supervise an innovative breeding ground for making algae biotechnological concepts economically attractive, useful for society and environmentally friendly.

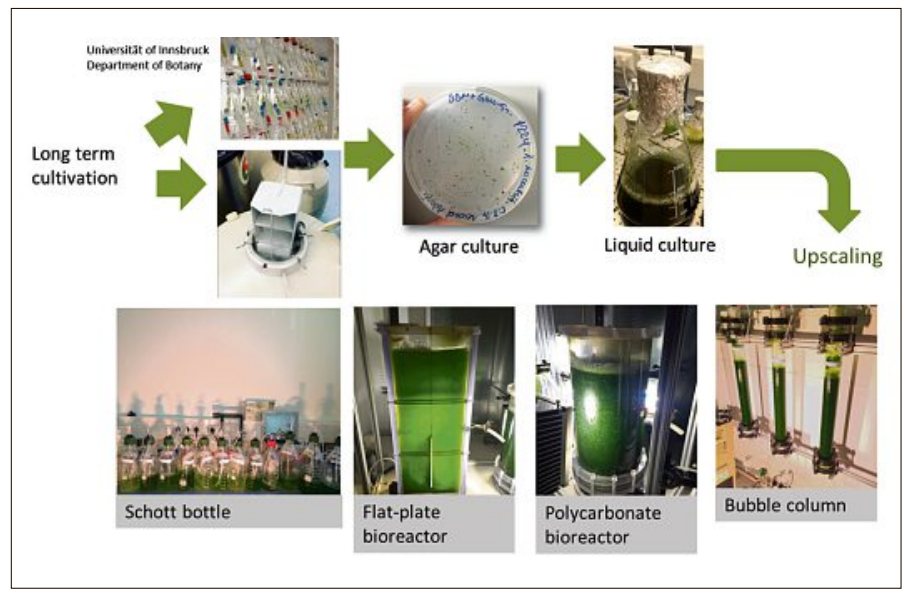

Maren Kruus: At the Management Center Innsbruck (MCl), researchers supervise an innovative breeding ground for making algae biotechnological concepts economically attractive, useful for society and environmentally friendly. (Source MCl)

The field of research at the MCI is the extraction of natural substances from algae. These activities range from the screening of algae collections and the development of cultivation screenings, via the optimisation of cultivation parameters and the screening of substances for pharmaceutical, cosmetic and food technological applications to the material and energetic recovery of biomass. "Since the MCI is part of the Open University Innsbruck concept, our partner of choice in this domain is the Institute of Botany at the University of Innsbruck. Its collection of algae dates back to the late 1950s and comprises about 500 strains, mostly from alpine areas of Central Europe, with a focus on terrestrial, airborne and lichen algae," says the researcher in Biotechnology Engineering. In 1974, the collection was expanded by incorporating the algae collection of Swiss botanist Wilhelm Vischer, a former lecturer at the University of Basel.

As important as maximising micro-algal growth rates may be, it must be kept in mind that many bioactive compounds are produced by stress-induced metabolic pathways and will not be synthesised in ideal culture conditions. This problem could be overcome by applying a two-stage process. First, sufficient biomass is 
produced under optimised growth conditions. Subsequently, the synthesis of the product of interest is induced by a specific stress treatment. "All in all, there is no approach that fits for all strains equally," Maren Kruus reminds us. "In order to increase microalgal biomass production or to stimulate metabolite synthesis, cultivation and fermentation strategies have to be optimised individually for every strain and product of interest."

During this study, the focus is mainly on culture purification and test strain cultivation optimisation. "Several screenings should be designed to effectively examine the individual growth patterns of the test strains," explains Maren Kruus. "The influence of light quality, trophic conditions as well as different nitrogen and carbon sources should be analysed. The results were used to establish a Design of Experiment (DoE) approach for further growth optimisation."

See CHIMIA 2015, 69, 362.

maren.kruus@mci.edu

www.mci.edu

\section{Active Antibiotic Monitoring for Personalised Treatment against Antimicrobial Resistance}

At FHNW Muttenz, the areas of interest of Senior Research Associate Natascha Kappeler are bacteria, resistance and in vitro diagnostics especially of infectious disease. As she notes, antibiotic resistance is predicted to kill more than 10 million people by 2050 with an economic burden of up to $\$ 100$ trillion. [1] "That's why our approach to combatting antibiotic resistance has to be multifaceted. Besides for instance drug discovery of novel antibiotics and understanding of resistance mechanisms, antimicrobial stewardship is critical to keep our precious antibiotics effective as long as possible. As both - over and under treatment - can fuel antimicrobial resistance, personalised medicine with individual dosing regimens is of upmost importance."

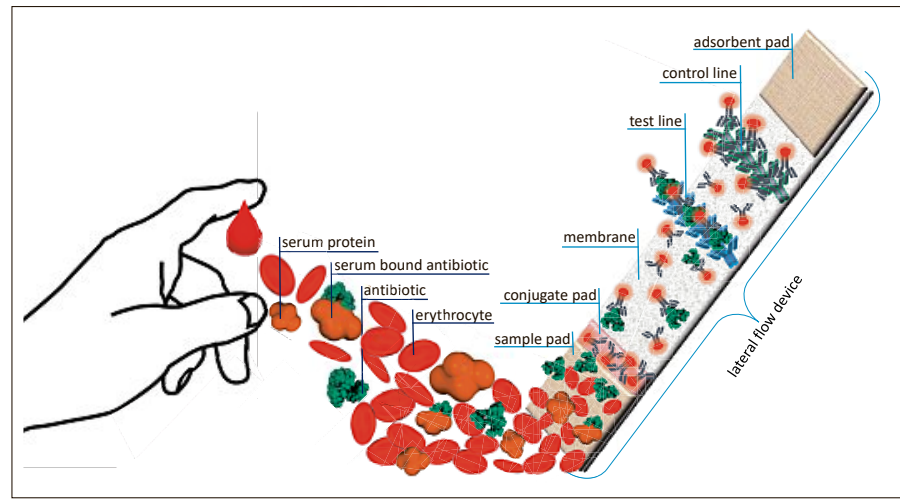

Natascha Kappeler: The schematic depicts a lateral flow device for therapeutic drug monitoring directly from a finger prick of blood that measures the free and active part of the antibiotic. The lateral flow device's mode of operation is direct with three different antibodies: one on the test line binding to the antibiotic, another one on the control line and the third one conjugated to a marker binding to the antibiotic and the control line's antibody. (Source Natascha Kappeler)

Many antibiotics are administered based on standard antibiotic dose response models relying on 'average' patient pharmacokinetics and pharmacodynamics. However, it is well recognised that this 'one dose fits all' approach fails to take into account underlying pathologies and phenotypic differences between individual patients, including metabolism and volumes of serum proteins. "Such serum protein volumes can be highly variable in elderly, pediatric, obese, oncology, immune-compromised and dialysis patients and since most therapeutic drug monitoring instruments only measure the total drug concentrations, doctors often have no choice but to just estimate the optimal dosing," explains Natascha Kappeler, whose training was carried out at University of Basel and University College London. "This is especially critical for drugs with narrow therapeutic windows, such as the antibiotic vancomycin - one of the last line treatments for multi-resistant bacteria, such as MRSA. Therefore, we are developing tests that measure fast, accurately and cost efficiently the active antibiotic concentration at the point-of-care, for example with paper based lateral flow devices."

natascha.kappeler@fhnw.ch www.fhnw.ch

[1] J. O’Neill, 'Tackling Drug-Resistant Infections Globally: Final Report and Recommendations', Review on Antimicrobial Resistance, 2016.

\section{Automatised Measuring Systems with Bacterial Biosensors}

Professor Martial Geiser, in charge of the research group 'Instrumentation and Control Systems at the HES-SO Valais/ Wallis', links biologists and engineers in an interdisciplinary approach to detect pollutants in water. "Detection of arsenic in water was part of the SAMS project initiated by UNIL Professor Jan van der Meer and funded by the Swiss National Science Foundation," explains the scientist. "It was carried out by making use of genetically engineered Escherichia coli cells which

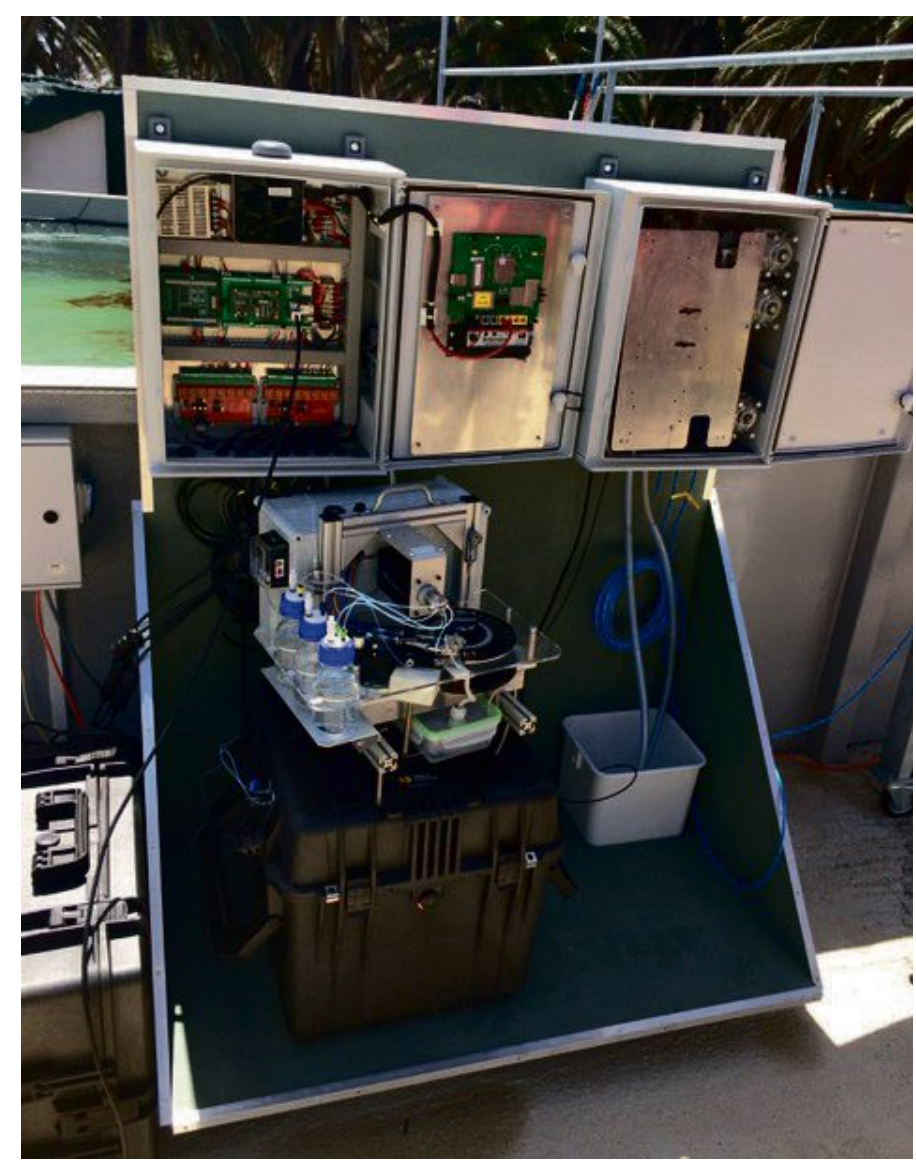

Martial Geiser: Device built at HES-SO for BRAAVOO. It will be mounted into a buoy and will perform measurements once a day using different genetically modified bioluminescent bacteria. The picture was taken in Messina (Italy) during the initial test. (Source Martial Geiser) 
produce fluorescent proteins when in contact with arsenic, creating an optical signal which can be measured."

"In the second project in which we participated-LiveSense of the Swiss Federal Research Program Nano-Tera - our goal was to build a semi-autonomous platform that supports cell-based sensing and sends results over the cellular network to a remote user," explains the physicist. "The bioreactors with cell models have been integrated to the environmental sensing system." The module can automatically adjust the osmolality of the water sample before being introduced in the bioreactor. The functionality with distance control by means of SMS was demonstrated.

Now all that's left to do is to incorporate a water 'robot' developed at EPFL, able to detect and track water pollutants in large surfaces of water. "This is the task of our Envirobot modules: they sample and measure relevant water quality parameters in surface water bodies by the incorporation of optical, physical, chemical and biological sensors," says Martial Geiser: "The robot is based on existing segmented anguilliform Amphibots, but with important adaptations in terms of energy use and efficiency, sensory decision programming, and communication possibilities." It was invented to perform autonomous surveying or autonavigation thanks to robotic engineers from the lab of Professor Auke Lispeert at EPFL.

Last but not least, the Framework Programme FP7 of the European Commission funded the project BRAAVOO, headed by Jan van der Meer, which concentrated on unmanned surveying vessels (USV) and buoys to carry 'Lab-on-a-Chip' modules for real-time monitoring of marine contaminants. Fluorescent bioreporter bacteria, immuno-sensors and algae provide the basis for biosensor instrument packs to be carried on such USVs.

In research, close co-operations between several partners always are a real challenge: "Biologists have to work with electrics, mechanics and robotics specialists and thus get to know other cultures even better; this chain of expertise guarantees us useful results and takes us a step forward in the future."

martial.geiser@hevs.ch

www.hevs.ch/en/rad-instituts/institute-of-systems-engineering/ pages-instituts/optoelectronics-9894

\section{On-line Monitoring and Control of Fed-batch Fermentations in Winemaking}

At ChemTech, the Institute of Chemical Technology at the Haute école d'ingénierie et d'architecture de Fribourg (HEIAFR), Dr Michal Dabros and his colleagues investigate the intensification of chemical processes. Based on their skills in chemistry and molecular characterisation, process development and scaleup as well as chemical engineering, automation and control, they want to create safer, environmentally cleaner and energy-saving processes. The techniques necessary to achieve these goals exist but they are often too complex and costly to be implemented industrially. "A recent project involved the implementation of a fully automated fed-batch wine fermentation process," recalls Michal Dabros. "The work was carried out in collaboration with the Haute Ecole de Viticulture et Oenologie de Changins and was reported in CHIMIA 2016, 70, 900." During the work, total sugars were monitored in real-time using mid-infrared spectroscopy and kept constant throughout the fermentation by an adaptive proportional-integral (PI) controller regulating the must feed flow rate. The major difficulties that were encountered included yeast sedimentation in the measurement flow-cell, as well as decreased controller robustness over time.

"The present follow-up study concentrated on resolving these issues and validating an improved methodology at the pilot scale," explains Michal Dabros, who is particularly interested

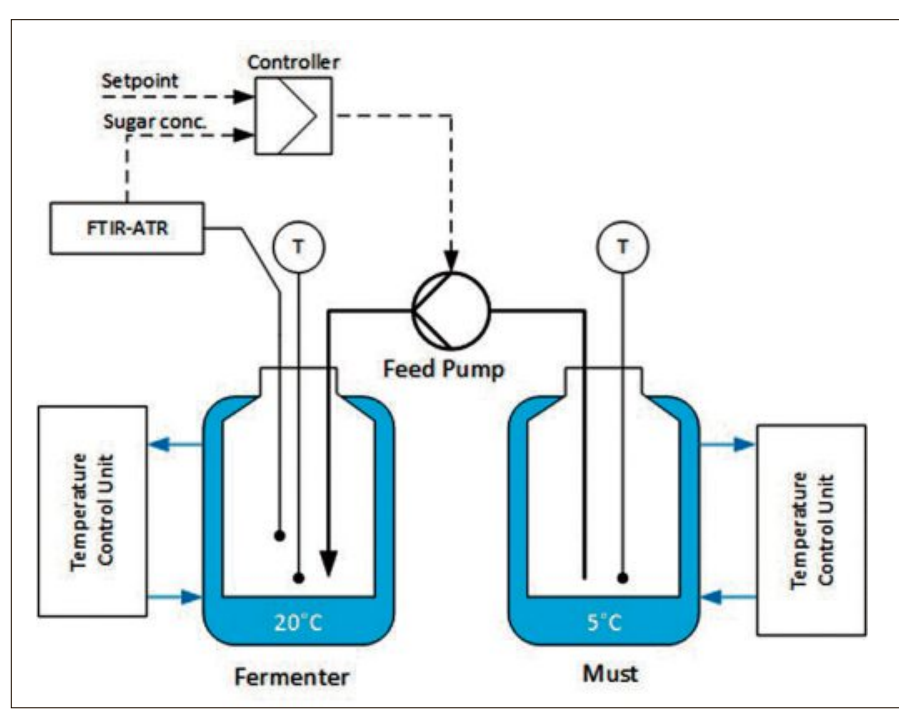

Michal Dabros: Process diagram of the experimental setup used for the monitoring and control of fed-batch wine fermentations. (Source Michal Dabros)

in on-line process monitoring and control. "Sugars and ethanol were monitored in-line using an in situ mid-infrared probe which not only solved the flow-cell clogging problem but also contributed to an increased sterility and non-invasiveness of the measurement technique. A simple model-predictive controller (MPC) was tested in parallel and proved to be more robust and stable than the adaptive PI controller applied previously." Over a period of four days, sugar concentrations were kept low and constant while ethanol levels rose as expected for a normal white wine fermentation.

The lecturer of Chemical Engineering takes stock: "It is believed that this approach significantly reduces cell stress and limits the production of unwanted secondary metabolites like acetic acid and acetaldehyde. Current work focuses on assessing this hypothesis and validating it on a larger production scale."

michal.dabros@hefr.ch

www.heia-fr.ch

\section{A Look Back}

"Setting up such an event certainly represents a lot of time and effort on the part of a whole group of dedicated people," says Professor Simon Crelier, coordinator of the Summer School 2017. "We are very happy that everything ran smoothly from the welcome reception on Sunday evening to the bus picking up the participants after the last presentation. The Summer School 2017 fulfilled its mission of promoting scientific exchanges between participants from highly diversified horizons, in a friendly and relaxed atmosphere. This would not have been possible without financial support from the Biotechnet and from the sponsors that kindly responded to our solicitation. Space is too limited to thank all who actively contributed but my colleagues and myself are very grateful that we rely on such a dedicated team. Long live the Summer School on advanced biotechnology, and see you all in Sicily next year!"

simon.crelier@hevs.ch

www.hevs.ch

Received: October 3. 2017 